\title{
The synthesis of adaptive multi-mode regulators based on combined control of the combined maximum principle.
}

\author{
Andrey Kostoglotov ${ }^{1}$, Sergey Lazarenko ${ }^{2}$, Vitaliy Losev ${ }^{2}$, and Igor Pugachev ${ }^{2},{ }^{*}$ \\ ${ }^{1}$ Rostov State Transport University, 344038, Rostov-on-Don, Russia \\ ${ }^{2}$ Don State Technical University, 344002, Rostov-on-Don, Russia
}

\begin{abstract}
The problem of synthesis of the optimal controls using nonlinear dynamic systems in general form is not yet analytically solved. Fundamental results of L.S. Pontryagin leads to programs of optimal control, and the solution to the synthesis problem can be obtained using complex constructions only in special cases. The implementation of such solutions implies the need to take into account the sliding modes, the regimes of frequent switching, the negative influence of interference, and so on. This was the reason for the development of methods for synthesizing systems of variable structure, which allow to increase the accuracy of control, eliminate undesirable modes, and counteract the influence of external and parametric disturbances. The results of the research showed that using the combined maximum principle leads to obtaining a general structure of quasi-optimal control for different classes of constraints and allows to provide parametric and structural adaptation to of the state and the parameters due to the possibility of complex implementation of control principles with respect to deviation and disturbance.
\end{abstract}

\section{Introduction}

The trend of development of modern controlled mechanical systems is due to the fact that these objects become essentially the multimode multipurpose systems of wide use. Therefore, the natural situation is when the control objective, the dynamic parameters of the control object and the external environment are all continuously changing. Linear control laws, as a rule, ensure the achievement of the goal of the solution for one mode of motion [1]. Meanwhile, the real controlled objects are often characterized by several modes of motion $[2,3]$. The theory of systems with variable structure leads to different controls obtained, each of that is constructed for the corresponding subsystem. As a result, we need to solve particular problems related to the choice of switching times, ensuring stability, reducing power consumption and increasing the performance $[4,5]$. Overcoming these drawbacks can be carried out by changing the switching surfaces, for example, using fuzzy control methods or artificial neural networks [5].

The basis for the procedures to obtain the equations of motion in sliding mode is the physical approach [3]. Its use for solving the problem of synthesis of regulators for the systems which dynamics satisfies the Lagrange equation of the second kind leads to a combined maximum principle [5-8]. The controls built on this base are characterized by the property to adapt to the change in the dynamic parameters of motion $[7,6]$ and by nonlinear structure of controls. The variety of solutions of the inverse problems is related to the different behavior of dynamic measures of motion on the switching hypersurface. Combination of the controls of the combined maximum principle makes it possible to achieve the stated synthesis goal for a set of motion modes that satisfy the Hamilton-Ostrogradskii principle [8].

The purpose of the work is to perform the synthesis of adaptive multi-mode regulators based on combined control of the combined maximum principle.

\section{Formulation of the problem}

The principle of combined control includes the implementation of control processes for deviation and disturbance (see Figure 1).

\footnotetext{
* Corresponding author: bakut_8536@mail.ru
} 


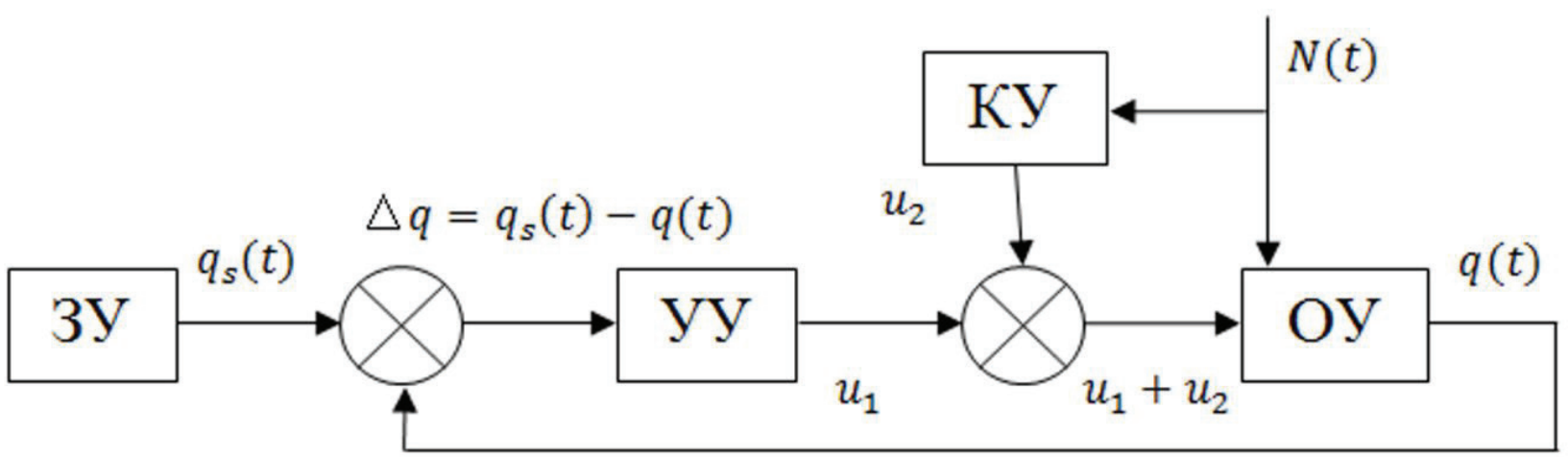

Fig. 1. The combined control

Where $3 y$ is the driver unit; $y Y$ is control unit; $K Y$ is compensating unit; $O Y$ is object of control; $q_{s}(t)$ is a given trajectory; $q(t)$ is the trajectory of the $O Y$ motion; $N(t)$ is external uncontrolled influence; $u(t)$ is the control action (control): $u_{1}(t)$ is control for deviation; $u_{2}(t)$ is control for disturbance ; $\Delta q(t)$ is deviation.

The structure of the combined management is the sum of the control actions $u_{s}$ defined on the sets $M_{1} M_{2}, \ldots M_{n}$. It consists of the elements of the merged sets

$$
\bigcup_{s=1}^{n} M_{s}=M_{1} \bigcup M_{2} \bigcup M_{n}
$$

In this case, the controls are the set of allowed impacts, following one after the other

$$
\bigcup_{s=1}^{n} u_{s}=\left\{u_{1} \in\left[0, t_{1}\right) \wedge u_{2} \in\left[t_{1}, t_{2}\right) \wedge \ldots\right\} .
$$

In addition the combined control can be defined on the intersection of sets

$$
\bigcap_{s=1}^{n} M_{s}=M_{1} \bigcap M_{2} \bigcap \ldots \bigcap M_{n} .
$$

In this case, the controls are the set of allowed impacts, performed simultaneously and taken with certain weights, i.e. complementing each other. Such combined controls are complementary:

$$
\bigcap_{s=1}^{n} u_{s}=\left\{u_{k} \in M_{1} \vee u_{k} \in M_{2} \vee \ldots\right\} .
$$

\section{The control of the pendulum oscillations in the presence of noise and disturbances}

Let us consider a pendulum, to which the control and the external action are applied (see Figure 2) [7, 8]. The notation in Fig. 2 is as follows: $I=m l^{2}$ is moment of inertia; $g$ is the gravity acceleration; $m$ is the mass; $l$ is the pendulum length; $u$ is the control; $P$ is the external influence.

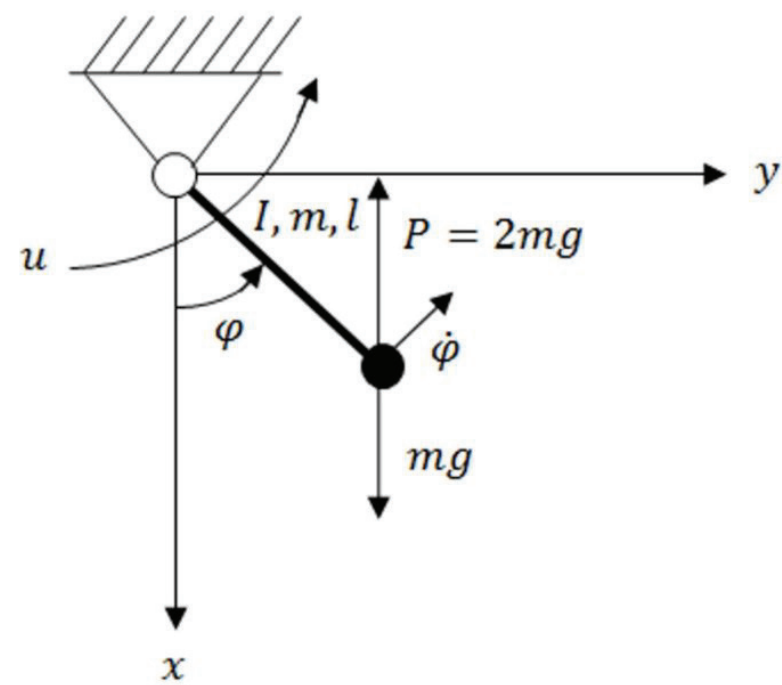

Fig. 2. The motion controls

where $I$ is moment of inertia of the body; $m$ is the mass; $l$ is the length; $u$ is the control; $P$ - is the external influence.

The state of the pendulum is determined by the generalized coordinates $q=\varphi, \dot{q}=\dot{\varphi}$

The kinetic energy $T$ and the momentum $p_{\varphi}=\frac{\partial T}{\partial \dot{\varphi}}$ of the moving pendulum are determined by formulas

$$
T=\frac{1}{2} I \dot{\varphi}^{2}, p_{\varphi}=I \dot{\varphi}
$$

The potential energy is given by expression

$$
\Pi=\operatorname{mgl}(1-\cos \varphi) \text {. }
$$

The work of generalized forces (control and disturbance) is

$$
A=\int_{0}^{q}(u-m g l \sin \phi+P l \sin \phi) d q .
$$

The target functional is as follows

$$
J=\frac{1}{2} \int_{0}^{t}(\phi-y(t))^{2} d t=\int_{0}^{t} F d t,
$$


where $y(t)=\phi+\xi(t)$ is the observation, $\xi(t)$ is stochastic process with known characteristics.

The Lagrange equation of the second kind for $P=0$ has the form

$$
\ddot{\phi}=-c \sin \phi+u, \quad c=\frac{g}{l} .
$$

\section{The control laws for classes of measurable and piecewise-continuous controls}

To obtain the conditions for a minimum of the target functional, we use the combined maximum principle, according to which we can write the extended Hamiltonian of the system as

$$
H=\lambda\left[T+\int_{q_{0}}^{q}(-c \sin \phi+u) d q\right]+F
$$

The generalized power is determined by function of the form

$$
\Phi=[\lambda(-c \sin \phi+u)+V] \dot{\phi} \rightarrow \max ,
$$

where $\lambda$ is the Lagrange multiplier,

$$
V=\frac{\partial F}{\partial \phi}=(\phi-y(t)) .
$$

The control from (16) can be defined as follows:

$$
u=\lambda^{-1}\left[\mu p_{\phi}-(V-\lambda \operatorname{csin} \phi)\right]
$$

where $\mu$ is synthesizing function.

The following variants to build the combined control are possible.

\section{The control is chosen from the class of bounded measurable functions}

The equation that defines the control switching line can be written as

$$
u_{0}=\lambda(-\sin \phi)+(\phi-y(t))=0 .
$$

Along this line the Hamiltonian is constant

$$
H_{0}=\lambda\left(T+\int_{0}^{\phi}-c \sin \phi d \phi\right)+F=\text { const } .
$$

The use of the operation of the Poisson bracket allows us to write:

$$
\frac{\partial H_{0}}{\partial p_{\phi}} \frac{\partial H_{0}}{\partial \phi}-\frac{\partial H_{0}}{\partial \phi} \frac{\partial H_{0}}{\partial p_{\phi}}=0,
$$

where $\frac{\partial H_{0}}{\partial p_{\phi}}=\lambda \phi ; \frac{\partial H_{0}}{\partial \phi}=(\phi-y(t)+\lambda \operatorname{csin} \phi)$;

$$
\frac{\partial u_{0}}{\partial p_{\phi}}=\mu ; \quad \frac{\partial u_{0}}{\partial \phi}=(1-\lambda \cos \phi),
$$

which yields

$$
\mu=\frac{-|\dot{\phi}| 1-\lambda \cos \phi \mid}{\lambda^{-1}|\phi-y(t)-\lambda \operatorname{csin} \phi|} .
$$

The law of the combined control with respect to deviation and disturbance has the form

$$
u=\lambda^{-1}\left[-\frac{|\dot{\phi}||\dot{\phi}| 1-\lambda \operatorname{ccos} \phi \mid}{\lambda^{-1}|(\phi-y(t))+\lambda \operatorname{csin} \phi|}-((\phi-y(t))-\lambda \operatorname{csin} \phi)\right] .
$$

The motion equation has the form

$$
\ddot{\phi}=\lambda^{-1}\left[-\frac{|\dot{\phi}||\dot{\phi}| 1-\lambda \cos \phi \mid}{\lambda^{-1}|(\phi-y(t))-\lambda \operatorname{csin} \phi|}-(\phi-y(t))\right] .
$$

\section{The control is chosen from the class of bounded functions}

Compensation for the perturbation can be performed using the forces superposition principle. Then we can put $u=u_{1}+u_{2}$, and here $u_{1}=c \sin \phi$. Hence

$$
\Phi=\left[\lambda u_{2}+(\phi-y(t))\right] \dot{\phi}=\max
$$

The conditions $u_{2}=\lambda^{(-1)}\left[\mu p_{\phi}-(\phi-y(t))\right]=0, u_{1}+c \sin \phi=0$ determine the switching line on which the Hamiltonian

$$
H_{0}=\lambda T+F=\text { const } \text {. }
$$

Using the Poisson brackets

$$
\begin{gathered}
\frac{\partial H_{0}}{\partial p_{\phi}}=\lambda \phi ; \quad \frac{\partial H_{0}}{\partial \phi}=(\phi-y(t)) ; \\
\frac{\partial u_{0}}{\partial p_{\phi}}=\mu ; \quad \frac{\partial u_{0}}{\partial \phi}=1
\end{gathered}
$$

provides the synthesizing function

$$
\mu=-\frac{|\dot{\phi}|}{\lambda^{-1}|(\phi-y(t))|} .
$$

The law of the combined control with respect to deviation and disturbance has the form 


$$
u=u_{2}+u_{1}=\lambda^{-1}\left[-\frac{|\dot{\phi}| \dot{\phi} \mid}{\lambda^{-1}|(\phi-y(t))|}-(\phi-y(t))\right] .
$$

The motion equation has the form

$$
\ddot{\phi}=\lambda^{-1}\left[-\frac{|\dot{\phi}||\dot{\phi}|}{\lambda^{-1}|\phi-y(t)|}-(\phi-y(t))\right] .
$$

\section{The control is chosen from the class of discontinuous piecewise-continuous and relay controls}

The piecewise constant equation

$$
H=\lambda\left(T+\int_{0}^{\phi}-c \sin \phi d \phi+\int_{0}^{\phi} u d \phi\right)+F
$$

The generalized power function

$$
\Phi=[\lambda(u-c \sin \phi)+(\phi-y(t))] \dot{\phi}=\max .
$$

To achieve the maximum we need to satisfy the following conditions:

$$
|u| \geq c|\sin \phi|=\text { const }, \quad u=|u| \operatorname{sign} \dot{\phi}
$$

The substitution of (25) into (23) gives

$$
H=\lambda[T-c(1-\cos \phi)+|u| \operatorname{sign} \dot{\phi}]+\frac{(\phi-y(t))^{2}}{2},
$$

which yields

$$
u=|u| \operatorname{sign}\left[-\frac{\left[\dot{\phi}^{2}+2 c(\cos \phi-1)+F\right] \operatorname{sign} \dot{\phi}}{2|u|}-(\phi-y(t))\right] .
$$

The motion equation has the form

$$
\ddot{\phi}=u \text {. }
$$

The piecewise-continuous control.

On the basis of the principle of the independence of the action of forces in the Hamiltonian, the generalized force can be determined as the sum of the forces

$$
Q=u-c \sin \phi
$$

Then

$$
H=\lambda\left[T+\int_{0}^{q} Q d q\right]+F
$$

and the generalized power function

$$
\Phi=(\lambda Q+(\phi-y(t))) \dot{\phi}=\max
$$

From the maximum condition it follows that

$$
|Q| \geq|\phi-y(t)|, \quad Q=k|\phi-y(t)| \operatorname{sign} \dot{\phi}
$$

where $k>1$.

Satisfying the maximum principle (2.19), the piecewise continuous control will have the form

$$
Q=k|\phi-y(t)| \operatorname{sign}\left[-\frac{\dot{\phi}^{2} \operatorname{sign} \dot{\phi}}{2}-\frac{(\phi-y(t))^{2} \operatorname{sign}(\phi-y(t))}{2}\right] .
$$

The motion equation has the form

$$
\ddot{\phi}=Q \text {, }
$$

the control

$$
u=Q+\operatorname{csin} \phi
$$

First we solve equation (32), then we solve equation (33).

The obtained basic solutions of optimal control can be extended on the basis of logical expressions

$$
u=\bigcup_{s=1}^{n} \varphi_{s}(a) u_{s}
$$

where $\phi_{S}(a)$ is logical function;

$a(\phi, \dot{\phi}, t)$ is function of the generalized coordinates and the time.

The combined control allows to eliminate the shortcomings of various devices by means of simple expression

$$
u=\sum_{s=1}^{n} a_{s} u_{s} \in \overline{G_{u}}
$$

where $a_{s}$ are weighting coefficients

$$
\sum_{s=1}^{n} a_{s}=1
$$

\section{The study of the effectiveness of the combined controls}

In the studies, we use the pendulum computational model (see Fig. 2).

The pendulum was subjected to loading by the reduced moment of gravity

$$
M_{0}=-c \cos \varphi, c=1 .
$$

and the control moment $u(t)$

In the process of controlling the pendulum, the measurements of the position of the pendulum are accompanied by noise with the characteristics $C K O=0,5, M M O=0,1$. Observation time 8; sampling period $\tau=0,01$. All values are dimensionless.

The results of the studies are shown in Fig. 3-4, where the phase portraits and the transient processes for laws (19), (21) and (26) are shown. Fig. 5 shows the nature of 
the combined control for the laws (19), (21) and (26), respectively.

The results of the studies confirm the effectiveness of the synthesized combined controls for obtaining the required quality of the process to control a nonlinear

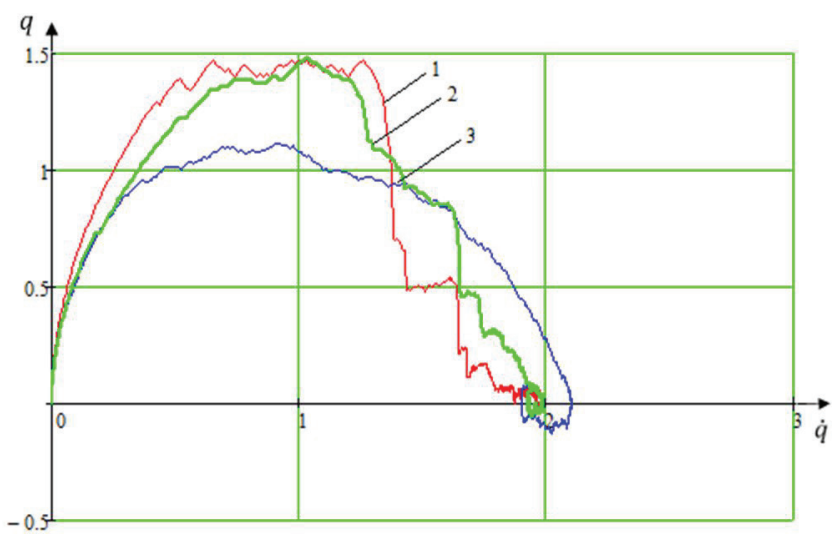

Fig. 3. The phase portrait: $1-\operatorname{control}(19) ; 2-\operatorname{control}(21) ; 3$ $-\operatorname{control}(26)$

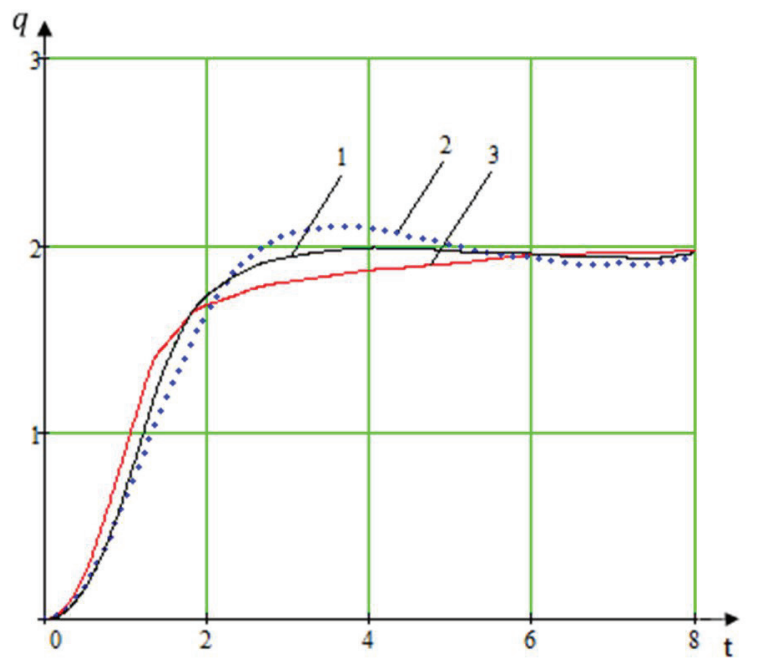

Fig. 4. The transient process: $1-\operatorname{control}(19) ; 2-\operatorname{control}(21)$; 3 - control (26)

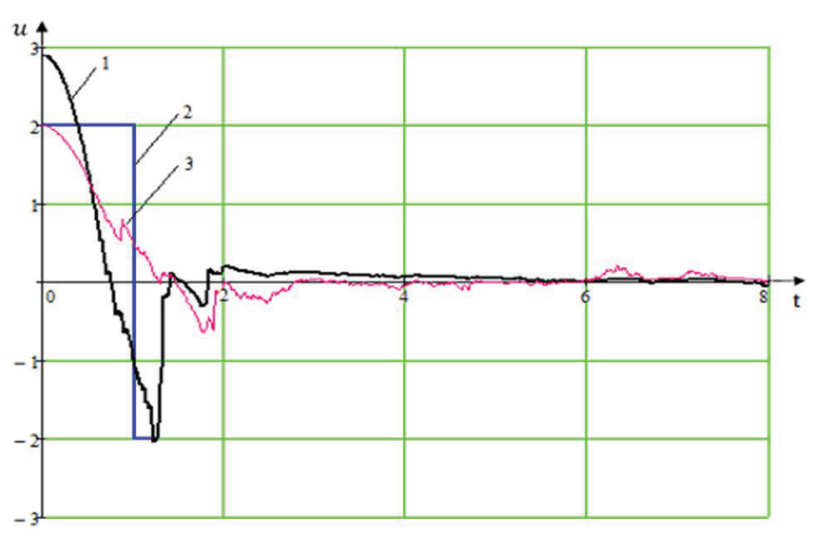

Fig. 5. The combined controls: 1 - control (19); 2 - control (21); 3 - control (26)

\section{Conclusions}

system - monotonic, aperiodic or oscillatory process with requirements for accuracy, overshoot, regulation time, etc.

On the basis of the theorem of the combined maximum principle, the basic laws to control the dynamic objects under the influence of external uncontrollable disturbances are obtained. The use of optimal laws to build the combined controls makes it possible: to exclude the chattering regimes generated by discontinuous (relay) controls in problems with the nonsmooth Hamiltonian; to ensure the stability of the object in an unbalanced state.

The paper has been accomplished with the support of Russian Federal Property Fund grants No. 15-08-03798.

\section{References}

1. R.A. Singer and K.W. Behnke, IEEE Transactions on Aerospace and Electronic Systems, no.1, $100-$ 110 (1971)

2. X.R. Li and V. Jilkov, IEEE Transactions on Aerospace and Electronic Systems, no.3, 1333-1364 (2003)

3. A.A. Kostoglotov, S.V. Lazarenko. Journal of Communications Technology and Electronics, no.2, $121-125$ (2017)

4. A.A. Kostoglotov, S.V. Lazarenko, Z.V. Lyaschenko. In Proc. 2017 20th IEEE International Conference on Soft Computing and Measurements, $568-571$ (2017)

5. S.V. Lazarenko, I.V. Pugachev, A.A. Kostoglotov, I.V. Deryabkin, and D.S. Andrashitov. In Proc. ICMSC 2017, 292 - 297 (2017)

6. A.A. Kostoglotov, I.V. Deryabkin, D.S. Andrashitov, S.V. Lazarenko, and A.A. Kuznetcov. In Proc. ICMSC 2017, 367-372 (2017) [in Russian]

7. A.A. Kostoglotov, S.V. Lazarenko, D.S. Andrashitov, and I.V. Deryabkin. [Online resourse] MATEC Web of Conferences. - 2016.

8. A.A. Kostoglotov, I.V. Deryabkin, S.V. Lazarenko, I.V. Pugachev, and A.A. Kuznetsov. In Proc. EWDTS 310 - 314 (2016) 